Mots. Les langages du politique

Trente ans d'étude des langages du politique

(1980-2010)

\title{
Questionner le discours avec Michel Foucault. Actualisations théoriques et actualité éditoriale
}

\section{Roselyne Ringoot}

\section{(2) OpenEdition \\ Journals}

Édition électronique

URL : https://journals.openedition.org/mots/19887

DOI : $10.4000 /$ mots. 19887

ISSN : 1960-6001

Éditeur

ENS Éditions

\section{Édition imprimée}

Date de publication : 30 novembre 2010

Pagination : 199-207

ISBN : 978-2-84788-235-3

ISSN : 0243-6450

\section{Référence électronique}

Roselyne Ringoot, « Questionner le discours avec Michel Foucault. Actualisations théoriques et actualité éditoriale », Mots. Les langages du politique [En ligne], 94 | 2010, mis en ligne le 06 novembre 2012, consulté le 28 avril 2022. URL : http://journals.openedition.org/mots/19887 ; DOI : https:// doi.org/10.4000/mots. 19887 


\section{Mots \\ Les langages du politique}

№ 94 novembre 2010

\section{Trente ans d'étude des langages du politique (1980-2010)}

ouvrage coordonné par Paul BACOT, Marlène Coulomb-GulLY, Jean-Paul Honoré, Christian Le BART, Claire Oger, Christian Plantin

\section{SOMMAIRE}

Paul Bacot, Marlène Coulomb-Gully, Jean-Paul Honoré, Christian Le Bart, Claire Oger, Christian Plantin

Le discours politique n'est pas transparent. Permanence

et transformations d'un objet de recherche

OUTILS ET ENJEUX DU DISCOURS POLITIQUE

Ruth Amossy, Roselyne Koren

Argumentation et discours politique

Christian Plantin

Argumentation-rhétorique. Les eaux mêlées

Caroline Ollivier-Yaniv

Discours politiques, propagande, communication, manipulation 
Marc Bonhomme

La caricature politique

Paul Bacot

Développement et diversification d'une onomastique politique

Ruth Wodak

The Discursive Construction of History. Brief Considerations

Henri Boyer

Les politiques linguistiques

\section{LIEUX DE LA PRODUCTION DU DISCOURS POLITIQUE}

Christian Le Bart

Parler en politique

Dominique Maingueneau

Le discours politique et son «environnement»

Alice Krieg-Planque, Claire Oger

Discours institutionnels. Perspectives pour les sciences

de la communication

Sophie Béroud, Josette Lefèvre

Le corpus syndical. Une expérience au long cours

Corinne Gobin, Jean-Claude Deroubaix

L'analyse du discours des organisations internationales.

Un vaste champ encore peu exploré

François de la Bretèque

Le retour de la parole politique dans le cinéma français

Jean-Claude Soulages

Vie et mort du citoyen cathodique

Jacques Guilhaumou

Les discours de la Révolution française.

Aperçu d'ensemble d'un trajet de recherche (1980-2009) 


\section{DISCIPLINES ET CHAMPS DE RECHERCHE POUR L'ÉTUDE DES LANGAGES DU POLITIQUE}

Philippe Braud

L'apport de la science politique à l'étude des langages du politique

Claire Blandin

L'apport de l'histoire des médias à l'étude des langages du politique

Jean-François Tétu, Bernard Lamizet

Les SIC et les langages du politique

Sylvianne Rémi-Giraud

Sémantique lexicale et langages du politique.

Le paradoxe d'un mariage difficile?

Marlène Coulomb-Gully, Juliette Rennes

Genre, politique et analyse du discours.

Une tradition épistémologique française gender blind

Johannes Angermüller

Analyser le discours politique en Allemagne (1980-2010)

Érik Neveu

L'apport de Pierre Bourdieu à l'analyse du discours.

D'un cadre théorique à des recherches empiriques

Roselyne Ringoot

Questionner le discours avec Michel Foucault.

Actualisations théoriques et actualité éditoriale

\section{ENTRETIEN}

Maurice Tournier

Mots et politique, avant et autour de 1980

La revue Mots. Les langages du politique encourage l'usage des rectifications de l'orthographe proposées par le Conseil supérieur de la langue française et approuvées par l’Académie (Journal officiel, $n^{\circ}$ 100, 6 décembre 1990). 


\section{Questionner le discours avec Michel Foucault. Actualisations théoriques et actualité éditoriale}

Depuis une quinzaine d'années, la publication de travauxinédits ou difficilement accessibles ${ }^{1}$ a renouvelé la présence éditoriale de Michel Foucault, au-delà de la réédition des ouvrages parus de son vivant tels que Les mots et les choses ou Surveiller et punir. Les désormais incontournables Dits et écrits, parus en 1994 dans la «Bibliothèque des sciences humaines » en quatre livres, ont été rassemblés en deux volumes sept ans plus tard dans la collection «Quarto » chez Gallimard. Ils regroupent des entretiens, des conférences, des textes produits en France ou à l'étranger, inscrits dans différents registres de discours : académique, militant, scientifique ou encore journalistique. Si la démarche consiste bien à rendre des textes disponibles, les éditeurs font néanmoins le constat suivant: «Nous savons que, quand bien même nos scrupules d'intervention minimale ont été constants, nous avons produit sous le nom de Michel Foucault quelque chose d'inédit. "² Les maisons d'édition Gallimard et Le Seuil s'engagent ensuite ensemble dans la publication des cours du Collège de France dispensés par Foucault dans le cadre de la chaire «Histoire des idées», dont il prend la charge en 19703. Le premier ouvrage de cette série paraît en 1997 : il s'agit du cours intitulé Il faut défendre la société. Sept volumes édités par des spécialistes 4 lui succèdent, dont, entre 1999 et 2003, Les anormaux, L'herméneutique du sujet, Le pouvoir psychiatrique. Deux ouvrages paraissent en 2004, Sécurité, territoire, population et Naissance de la biopolitique, alors que Le gouvernement de soi et des autres / et Le gouvernement de soi et des autres II. Le courage de la vérité sortent respectivement en 2008 et 2009 . Les textes, qui respectent le style oral de l'exercice, sont établis à partir d'enregistrements sonores (parfois peu performants) et de manuscrits.

Les Dits et écrits, tout comme les Cours au Collège de France, représentent un enjeu majeur du point de vue de l'intertextualité foucaldienne, car ils

IUT Lannion, CRAPE

ringoot.rosy@laposte.net

1. Mis à part au Centre Foucault.
2. Daniel Defert, François Ewald, Jacques Lagrange éd., Dits et écrits I, p. 12, reprenant la présen-
tation de l'édition de 1994 .
3. Jusqu'à son décès en 1984 .
4. Voir en fin d'article les références complètes de ces publications. 
instaurent d'autres pistes de lecture que celles des livres publiés par Foucault en personne. Ces derniers s'en trouvent éclairés, contextualisés, mis en dialogues par des textes jusqu'alors disséminés ou indisponibles. Bien évidemment, cette intertextualité ne repose pas uniquement sur une simple mise à disposition de travaux. Elle est aussi redevable du traitement éditorial qui implique les actions de sélection, d'organisation, de présentation, de mise en forme, etc. La responsabilité des éditeurs paraît d'autant plus prégnante lorsqu'il s'agit de publier à titre posthume, surtout quand on sait que Foucault ne le souhaitait pas. Daniel Defert, dépositaire des notes et manuscrits de son ancien compagnon, explique sa démarche et son interprétation de «la catégorie des publications posthumes » : "Les Dits et écrits n'y entrent pas, puisqu'on a retenu uniquement les textes qui étaient parus ou en cours de parution au moment de sa mort; les cours, eux, avaient eu une forme de publicité orale. Les aveux de la chair c'est un autre problème.» (Defert, 2007) Ce dernier, quatrième tome de l'Histoire de la sexualité, reste sous le sceau de l'interdiction testamentaire.

Considérés dans leur singularité car révélant des approches propres et autonomes ou envisagés dans une perspective intertextuelle, ces textes, notamment les derniers cours du Collège de France, créent les conditions matérielles de nouvelles lectures et de nouvelles appropriations. Poser l'hypothèse d'un lien fort entre les apports de Foucault dans les recherches contemporaines et la configuration éditoriale des deux dernières décennies paraissait alors légitime. Choisissant par ailleurs de restreindre le champ des actualisations de Foucault à celles qui concernent la question du ou des discours, cette contribution veut donc éprouver l'hypothèse de l'influence des publications récentes sur les travaux liés à l'étude des langages du politique telle que promue par la revue Mots. Les langages du politique. Or, la piste du discours qui est ici privilégiée amène à relativiser l'hypothèse de départ, ou pour le moins, à la nuancer. Si les effets de l'actualité éditoriale sont facilement perceptibles dans le champ de la science politique, et plus particulièrement autour de la notion de "gouvernementalité », ils paraissent plus complexes à évaluer dans le domaine de l'analyse de discours. Les références y concernent l'époque des Mots et les choses (1966), L'Archéologie du savoir (1969) ou encore L'ordre du discours5, donc tout autant ces livres eux-mêmes que des textes contemporains indexés dans Dits et écrits (le premier volume dans la collection «Quarto ».) Les publications récentes des cours du Collège de France semblent avoir peu d'impact sur les travaux des spécialistes du discours, probablement au motif qu'elles n'interrogent pas directement ni exclusivement la dimension discursive. Fautil en déduire que la réception des travaux de Foucault diffusés dernièrement obéit à la cartographie des disciplines académiques et des objets traités et

5. Leçon inaugurale du Collège de France prononcée le 2 décembre 1970 et publiée chez Gallimard l'année suivante. 
construits, plus que ce ne fut le cas de son vivant? Cette contribution insinuerait alors que la question du discours est aussi présente dans des textes qui ne lui sont pas proprement dédiés, et que leur lecture peut être féconde dans la mesure où le gouvernement de soi et des autres qui occupe Foucault à partir des années soixante-dix n'obère pas l'articulation aux matérialités discursives. Mais voyons dans un premier temps les apports de Foucault aux études spécialisées sur le discours, avant de considérer ceux qui alimentent l'étude de l'action publique et dont on peut tirer parti en termes d'approches discursives.

\section{Travailler la discursivité}

Les apports de Foucault à l'analyse du discours sont indissociables de la théorie des formations discursives développée dans les années soixante/soixantedix. Dominant à l'époque inaugurale de l'analyse de discours française, l'usage de la formation discursive connaît un reflux à partir des années quatre-vingt ${ }^{6}$. Dans la décennie qui suit, la notion donne lieu à des exploitations contrastées, entre homogénéité et hétérogénéité discursive. Pour Jean-Michel Adam (1997), un journal, un parti politique, un syndicat ou une église sont autant de formations discursives susceptibles de fonctionner dans un régime d'interdiscursivité. Jacques Guilhaumou, qui a la particularité d'avoir exposé son rapport problématique à la formation discursive dont il a été l'un des penseurs, réinvestit la notion qu'il avait un temps abandonnée. Celle-ci est convoquée dans son travail sur la «parole des exclus» mené dans la décennie quatre-vingt-dix, selon de nouvelles perspectives. Revenant sur son actualisation conceptuelle sous forme de bilan critique en 2004, l'historien et linguiste opère une inflexion qui oriente l'usage de la formation discursive en fonction du sujet empirique, «à la charnière de l'usage des mots, donc de leur utilité, et de leur lien à la pensée, donc de leur vérité» (Guilhaumou, 2004).

Plus récemment, la focalisation sur des notions afférentes à celle de la formation discursive donne matière à des exploitations différenciées appliquées aux langages du politique et du médiatique. Dominique Maingueneau revient sur cette catégorie notionnelle dans un article datant de 2005, en l'associant à celle de "parcours », qui renvoie aux travaux d'Alice Krieg-Planque sur la formule «épuration ethnique» et à ceux de Sophie Moirand sur la «mémoire discursive ». Néanmoins, parcours et formation discursive, en tant qu' « unités non topiques », « attirent [...] plus facilement la suspicion » que la classe des "unités topiques" comprenant les genres de discours et les registres communicationnels» (Maingueneau, 2005). Pour Juliette Rennes et Pierre Fiala, c'est la notion

6. Entrée «formation discursive » dans le Dictionnaire d'analyse du discours (Charaudeau, Maingueneau, 2002). 
de «dispositif» qui est retenue et convoquée dans leur travail sur la politique migratoire sécuritaire européenne, inscrit au sein d'un programme collectif genevois dont ils rendent compte en 2006. L'ensemble des contributions est présenté au regard « de la construction des réseaux sémantiques et formels, à la fois hétérogènes, transdisciplinaires, historiquement actifs, objets permanents de controverses et d'enjeux» (Fiala, Rennes, 2006). Cette actualisation du «dispositif» foucaldien se réfère aux Dits et écrits.

On remarque aussi la résurgence de la notion d'auteur chez Dominique Maingueneau qui, dans la continuité de sa recherche sur l'ethos discursif, interroge «l'image d'auteur» développée actuellement en analyse de discours. Il renouvelle ainsi l'approche de l'« auctorialité» au regard du « régime numérique» des textes produits sur Internet, en se rapportant à la notion d'œuvre et d'auteur traitée par Foucault à la fin des années soixanter. Finalement, dans la revue Mots. Les langages du politique (et plus généralement en analyse de discours), les travaux actualisant Foucault se réfèrent plus aux Dits et écrits qu'aux publications des cours du Collège de France. Une contribution contredit malgré tout ce constat, car dans leur travail qui porte sur l'usage des termes cité, civilité, urbanité, citoyenneté et espace public, Philippe Genestier, Samira Ouardi et Juliette Rennes utilisent la théorisation de «l'État pasteur» en se fondant sur les ouvrages de 2001 et 2004. Publiée dans Mots. Les langages du politique en 2007, cette analyse des politiques sociales françaises démontre comment les politiques de la ville et les discours localistes (Genestier, Ouardi, Rennes, 2007) ont reformulé les problèmes politiques et sociaux sous le gouvernement Jospin.

\section{Autour de la "gouvernementalité»"}

Dans les cours du Collège de France donnés de 1977 à 1979 et publiés en 2004, Sécurité, territoire, population et Naissance de la biopolitique, édités par Michel Sennelart, gouvernementalité s'avère un mot crucial et une question centrale. La gouvernementalité est un néologisme inventé par Foucault qui vient ainsi élargir le champ lexical des langages du politique. Par cette désignation, il saisit et signifie la matérialité étatique en marquant la volonté de s'attacher aux pratiques de l'État plus qu'à sa théorie ou à son essence. Le gouvernement est pour lui un substantif fonctionnant comme un verbe transitif direct : le gouvernement des autres ou encore le gouvernement de soi. À l'opposé de la gouvernance qui peut être bonne, mauvaise, mondiale ou locale, la gouvernementalité exclut tout épithète. II s'agit d'un concept qui renvoie à

7. Maingueneau, 2009. Maingueneau se réfère à la notion d'œuvre traitée par Foucault dans L'archéologie du savoir et à celle d'auteur traitée dans la conférence «Qu'est-ce qu'un auteur? », dont il indique la publication dans le Bulletin de la Société française de philosophie en 1963 et sa reprise dans Dits et écrits de 1994 . 
l'effectuation plus qu'à l'intention, à la concrétisation plus qu'au programme. Reprendre à son compte le concept de gouvernementalité aujourd'hui est une manière d'élargir le champ des empiries des actions et des techniques de gouvernement, de repérer le degré d'étatisation de la société. C'est aussi mesurer les paradoxes du libéralisme contraignant qui implique plus de liberté et de bien-être, par le biais des technologies de pouvoir d'autant plus efficaces qu'il faut « gouverner moins».

Pierre Lascoumes a particulièrement essaimé sur cet apport de Foucault, en nom propre ou en collaboration, notamment avec Patrick Legalès (Lascoumes, Legalès, 2007; Lascoumes, Legalès éd., 2004) ou Pascale Laborier (Laborier, Lascoumes, 2005). La théorisation du rapport gouvernant/gouverné est liée au concept d'instrumentation utilisé dans des travaux empiriques portant sur l'action publique. Bien que non centrale, la question du discours se pose de manière stimulante par le biais de l'information et de la communication considérées en tant qu'instruments. Instruments qui organisent le rapport politique de "démocratie au public » et qui légitiment «l'explicitation des décisions et la responsabilisation des acteurs» (Lascoumes, 2004). La gouvernementalité revisitée à l'aune des politiques publiques est aussi actualisée dans l'approche des problèmes publics et en sociologie des controverses. La «mise en visibilité » et la « mise en politique » qui charpentent l'analyse des processus de construction des problèmes publics, sans se référer obligatoirement à Foucault, sont susceptibles de lui devoir beaucoup par intermédiation. Pour Pierre Lascoumes, «le dernier "tour de main" serait de chercher à rendre gouvernables les situations sociales problématiques (controversées ou conflictuelles) en organisant, non pas leur transparence, mais leur mise en débat et peut être plus fondamentalement leur mise en discours» (Lascoumes et al., 1997), position qui s'appuie sur l'articulation orthodoxie/orthologie 8 proposée par Foucault dans son cours de 1975-1976 (Foucault, 1997). Les forums délibératifs, les arènes publiques, la désignation, les processus de catégorisation et les catégories floues renouvellent l'approche et le questionnement des matérialités discursives et de la dispersion ordonnée foucaldienne. Si l'on considère plus particulièrement l'année 2004, lors de laquelle est commémoré le vingtième anniversaire de la mort de Foucault, des colloques donnant lieu à des numéros spéciaux de revues, notamment Vacarme 9 et Le Portique ${ }^{10}$,

8. "L'orthodoxie classique porte sur le contrôle du contenu des énoncés par référence à un dogme plus ou moins fondé. L'orthologie concerne le contrôle de la régularité des formes d'énonciation. Elle correspond à une disciplinarisation interne des savoirs et à la mise en place de procédures attachées autant au mode de production et de circulation des énoncés qu'à leur contenu lui-même.» (Foucault, 1997, p.15-16)

9. En ligne [URL: http://www.vacarme.org/rubrique105.html]. Site consulté le 22 juillet 2010.

10. En ligne [URL : http://leportique.revues.org/index601.html]. Le numéro est introduit par JeanFrançois Bert, un jeune sociologue. Site consulté le 22 juillet 2010. 
ou des livres ${ }^{11}$, attestent un intérêt renouvelé pour Foucault en sciences sociales, y compris chez de jeunes chercheurs.

\section{Discontinuités à propos du discours}

Fondamentale dans les années initiales de l'analyse de discours française, la notion de formation discursive est alors travaillée par des linguistes, historiens et sociologues (Conein et al., 1984, p. 25-30) rassemblés autour de Michel Pêcheux, dont les objets privilégiés sont les idéologies et le politique. La phase de disciplinarisation de l'analyse de discours s'accompagne ensuite d'une tendance à figer le discours dans une pureté énonciative et une unité d'acteurs allant à l'encontre de la complexité des fonctionnements discursifs appréhendée par Foucault. Parallèlement, à la même époque, les usages se raréfient. Depuis quelques années, on assiste à une résurgence de l'influence foucaldienne dans des travaux qui actualisent des concepts afférents à celui de la formation discursive, puisant éventuellement dans les publications (presque) posthumes. Michel Foucault est le penseur du décentrement de l'objet et du déplacement théorique, posture se prêtant peu à la pérennisation d'un courant ou d'une école. C'est pourquoi il est difficile de réduire son action à un simple «apport » à l'étude du discours (depuis la création de la revue Mots. Les langages du politique) qui serait le legs de la formation discursive. Le retour sur ce concept s'effectue selon des appropriations qui assument une distance vis-à-vis de lui et selon des détours par d'autres notions qui lui sont complémentaires mais qui s'en éloignent éventuellement. Foucault se présentait volontiers comme un pourvoyeur d'outils à piocher dans ses textes, et finalement, les utilisations de ses concepts s'avèrent plus convaincantes quand elles assument une actualisation critique et distanciée. Il revient au chercheur de trouver sa propre opérationnalité à partir des hypothèses que Foucault délivre «sous une forme très condensée » (Lascoumes, 2004). Ses termes, bricolés ou sémantisés de manière alternative, ouvrent des possibles théoriques et méthodologiques à mettre en place, comme le prouvent les spécialistes de la gouvernementalité et de l'instrumentation.

La dispersion est un autre mot-notion permettant de questionner une pratique discursive telle que celle du journalisme. Le point de vue défendu ici pointe que la dispersion, qui implique une approche du journalisme centrée sur le discours, rentre néanmoins difficilement dans les cases discipline/objet de l'analyse du discours journalistique stricto sensu (Ringoot, 2010). En revisitant le concept de dispersion (Ringoot, Utard, 2009), il s'agit de questionner les métacatégorisations des discours sociaux (politique, journalistique,

11. Voir notamment Meyet, Naves, Ribemont, 2005. Les coauteurs étaient alors doctorants en science politique. En sciences de l'information et de la communication, voir Chevalier, Loneux, 2006. 
scientifique, etc.) sans perdre de vue que les acteurs sociaux produisent leur identité en se fondant sur la distinction de leurs pratiques discursives. Dans le domaine du journalisme, placer la perspective de recherche sur les croisements, imbrications et ajustements discursifs permet d'observer les écarts entre ce qui relève de la légitimité sociale construite par les journalistes sur le mode oppositionnel, et ce qui relève des arrangements discursifs subis, négociés ou sollicités. Cette démarche qui veut questionner le discours saisi entre coercition et dispersion s'inspire de L'archéologie du savoir et des Dits et écrits axés sur la discursivité, mais la réflexion autour de la gouvernementalité, bien que ne prenant pas le discours comme objet central, ouvre des pistes quant à l'information et la communication. Sur un autre plan, les régimes de vérité sur lesquels Foucault revient à la fin de sa vie offrent des perspectives à explorer dans le cadre des récits et des discours, particulièrement en journalisme. Les modes de véridiction liés à la teknê, au savoir-faire ou à la parresiâ, « dire vrai » associé à une prise de risque ${ }^{12}$, suscitent une réflexion qui, sans prétendre à une nouveauté fondamentale, mériterait un approfondissement.

Au regard de cette rétrospective circonscrite, les coupures présupposées entre les périodes foucaldiennes - particulièrement celles qui distinguent littérature/discours, politique/pouvoir et éthique/subjectivité (Revel, 2005) et autour desquelles s'organisent les postérités produites par les différents terreaux scientifiques - sont vivaces. Mais elles n'épuisent pas la possibilité d'autres lectures éventuellement moins conformes aux découpages disciplinaires intériorisés et extériorisés, ou à la sectorisation attendue par objet de recherche. C'est dire autrement qu'une investigation des «derniers » Foucault à partir d'une approche centrée sur les pratiques discursives reste à faire, dans la mesure où les derniers cours au Collège de France n'échappent ni à l'archéologie du discours, ni à la généalogie des pratiques qui constituent le fil directeur de la pensée de Michel Foucault.

\section{Références}

ADAM J.-M., 1997, «Unités rédactionnelles et genres discursifs. Cadre général pour une approche de la presse écrite», Pratiques, nº 94, juin.

Charaudeau P., Maingueneau D., 2002, Dictionnaire d'analyse du discours, Paris, Le Seuil.

12. «Il faut pour qu'il y ait parrêsia que, en disant la vérité, on ouvre, on instaure le risque de blesser l'autre, de l'irriter, de le mettre en colère et de susciter de sa part un certain nombre de conduites qui peuvent aller jusqu'à une extrême violence. » Foucault, 2009, p. 12. 
CheValier Y., Loneux C., 2006, Foucault à l'œuvre. Deux années de lecture foucaldienne dans un laboratoire de SHS, Fernelmont, Éditions modulaires européennes et intercommunications (Échanges).

Conein B., Guilhaumou J., MALDidier D., 1984, «L'analyse de discours comme contexte épistémologique », Mots. Les langages du politique, nº 9, octobre, p. 25-30.

DEFERT D., 2007, "“Je crois au temps..." Daniel Defert légataire des manuscrits de Michel Foucault», propos recueillis par G. Bellon, Recto/Verso, $n^{0} 1$, juin, en ligne [URL : http://www.revuerectoverso.com]. Site consulté le 22 juillet 2010.

Genestier P., OuArdi S., Rennes J., 2007, «Le paradigme localiste au secours de l'action publique démocratique», Mots. Les langages du politique, n 83, mars.

Guilhaumou J., 2004, "Où va l'analyse de discours? Autour de la notion de formation discursive», Texto!, en ligne [URL : http://www.revue-texto.net/Inedits/Guilhaumou_AD.html]. Site consulté le 22 juillet 2010.

FiALA P., RENNES J., 2006, " Notes sur une participation à une expérience de recherche », à propos des Actes du Colloque international de Genève, septembre 2004 (M.-

C. Caloz-Tschopp éd.), Mots. Les langages du politique, no 81, juillet, p.55-60.

LABORIER P., LASCOUMES P., 2005, "L'action publique comprise comme gouvernementalisation de l'État», Travailler avec Foucault. Retour sur le politique, S. Meyet, M.-C. Naves, T. Ribemont éd., Paris, L'Harmattan, p. 37-62.

LASCOUMES P. et al., 1997, Information, consultation, expérimentation. Les activités et les formes d'organisation au sein des forums hybrides, Points de vue de P. Lascoumes, M. Callon, Y. Barthes, Séminaire du programme «Risques collectifs et situations de crise ", Actes de la $8^{e}$ séance, Grenoble, CNRS.

LAscoumes P., Legalès P., 2007, Sociologie de l'action publique, Paris, Armand Colin.

LASCOUMES P., 2004, "La gouvernementalité. De la critique de l’État aux technologies du pouvoir », Le Portique, en ligne [URL : http://leportique.revues.org/index625. html]. Site consulté le 22 juillet 2010.

LASCOUMES P., LegALÈs P. éd., 2004, Gouverner par les instruments, Paris, Presses de Science Po.

Maingueneau D., 2005, "L'analyse du discours et ses frontières", Marges linguistiques no 9, p. 73-74, en ligne [URL : http://www.revue-texto.net/1996-2007/marges/ marges/Documents\%20Site\%201/o0_ml092005/00_mlo92005.pdf]. Site consulté le 22 juillet 2010.

Maingueneau D., 2009, «Auteur et image d'auteur en analyse du discours », Argumentation et analyse de discours, nº3, en ligne [URL : http://aad.revues.org/index66o. html]. Site consulté le 22 juillet 2010.

MeYet S., NAVes M.-C., RiBemont T., 2005, Travailler avec Foucault. Retour sur le politique, Paris, L'Harmattan.

ReVEL J., 2005, Michel Foucault. Expériences de la pensée, Paris, Bordas.

RING OOT R., UTARD J.-M., 2005, Le journalisme en invention, Rennes, Presses universitaires de Rennes (Res publica).

RINGOOT R., UTARD J.-M., 2009, Les genres journalistiques. Savoirs et savoir- faire, Paris, L'Harmattan (Communication et civilisation).

RINGOOT R., 2010, «Le statut encombrant du discours dans l'étude du journalisme», 
Figures sociales du discours. Le «discours social» en perspective, A. Tavernier et al. éd., Lille, Université de Lille 3.

\section{Fuvres de Michel Foucault}

1966, Les mots et les choses, Paris, Gallimard.

1969, L'archéologie du savoir, Paris, Gallimard.

1971, L'ordre du discours, Paris, Gallimard.

2001, Dits et écrits, t. I, 1954-1975, t. II, 1976-1988, Paris, Gallimard (1994, 4 vol., même

éditeur).

Cours au Collège de France (CCF) édités sous la direction générale de François Ewald et Alessandro Fontana, Paris, Gallimard / Le Seuil / EHESS :

1997, CCF 1975-1976. Il faut défendre la société, M. Bertani, A. Fontana éd.

1999, CCF1974-1975. Les anormaux, V. Marchetti, A. Salomoni éd.

2001, CCF1981-1982. L'herméneutique du sujet, F. Gros éd.

2003, CCF 1973-1974. Le pouvoir psychiatrique, J. Lagrange éd.

2004, CCF1977-1978. Sécurité, territoire, population, M. Sennelart éd.

- CCF1978-1979. Naissance de la biopolitique, M. Sennelart éd.

2008, CCF 1982-1983. Le gouvernement de soi et des autres I, F. Gros éd.

2009, CCF 1983-1984. Le gouvernement de soi et des autres II. Le courage de la vérité,

F. Gros éd. 